\title{
A Much Advanced and Efficient Lane Detection Algorithm for Intelligent Highway Safety
}

\author{
Prof. Sachin Sharma ${ }^{1}$ and Dr. D. J. Shah ${ }^{2}$ \\ ${ }^{1}$ Department of Electronics \& Communication, GTU, Ahmedabad, India \\ sharma.f@gmail.com \\ ${ }^{2}$ Department of Electronics \& Communication, GTU, Ahmedabad, India \\ djshahelcit.org
}

\begin{abstract}
This paper presents a much advanced and efficient lane detection algorithm. The algorithm is based on (ROI) Region of Interest segmentation. In this algorithm images are pre-processed by a top-hat transform for de-noising and enhancing contrast. ROI of a test image is then extracted. For detecting lines in the ROI, Hough transform is used. Estimation of the distance between Hough origin and lane-line midpoint is made. Lane departure decision is made based on the difference between these distances. As for the simulation part we have used Matlab software.Experiments show that the proposed algorithm can detect the lane markings accurately and quickly.
\end{abstract}

\section{Keywords}

Hough transform, Top-Hat transform, lane detection, lane departure, ROI Segmentation.

\section{INTRODUCTION}

With the help of offered machine vision algorithms, dozens of processors control every performance aspect of today's automobiles which are rising exponentially. In the future, vehicles tend to be more intelligent and shall assist the driver both concerning comfort and safety. Several facilities are being offered under Advanced Driver Assistance Systems (ADAS) like night vision assistance, lane departure warning system (LDWS), pedestrian detection system (PDS), smart airbags, cruise control, etc. As the reliability and the performance of the algorithms have been significantly improved due to the increasing performance of computers, vision systems have been acknowledged in the automatic control community as a powerful and versatile sensor to measure motion, position and structure of the environment. If efficient algorithms are developed for such modern vision systems, then the performance of the system will certainly improved to large extent. With increase in the challenges in identifying the road lanes, robust algorithms must be used to mitigate the problems of poor lane detection, less efficiency poor performance under different traffic and environmental conditions. Many time road lanes are fade and not visible. 


\section{PROBLEM ADDRESSED}

With increasing challenges in the identification of road lanes, robust algorithms must be used to mitigate the problems of poor lane detection, less efficiency, poor performance in traffic and different environmental conditions. Many time road lanes are fade and not visible. Two-lane, three lane, and four - lane roads are present in many cities of the developed and under-developed countries. Such factors are becoming obstacles in identifying the road lane for Lane Departure Warning Systems (LDWS). Especially, when multiple lanes are present on a road, the detection algorithm may identify all these lanes due to viewing angle of camera inside a car or vehicle. On urban highways, multiple entry and exit points are present with relatively small distances between adjacent entry and exit points. This scenario explains the presence of various lane markings on urban roads. For LDWS, these detected lane edges may lead detection algorithm towards complexity and inaccuracy. Also, while giving departure warning, multiple lane boundaries may give false warnings. During the processing of lane departure, time to lane crossing (TLC) parameter may be affected.

According to the survey carried out by National Highway Traffic Safety Administration of US, $43 \%$ of the total traffic accident casualties are the results of the abnormal lane switching/departure on the road, which is also the major cause of the traffic accident in the list [1]. In the previous studies of the Driver Assistance System (DAS), a much powerful computing machine and large size memory are required to carry out the calculation of the computer vision and graphic processing algorithms [2], [3]. There are articles contributed to the studies and methods of lane recognition, such as the stereo vision system [4], [5] which transform the image coordinate system back to the real world coordination. Then, the method is applied to identify the lane markings and remove other irrelevant objects in the image. To improve the performance, it was proposed using curvature method only in the far end of the image but adopting the straight line pattern in the near end to identify the lane markings in order to reduce the time required for identification [6]. Many approaches have been applied to lane detection, which can be classified as either feature-based or model based [7], [8]. Hsiao et. Al. presents lane departure algorithm based on spatial and temporal mechanism [9]. But this approach suffers from poor illumination problem. In [10]-[11], occlusion handling algorithm for lane tracking is presented. But is has a limitation of low computational speed.

\section{ABOUT THIS PAPER}

In this paper, effective ROI is considered as a first step of algorithm processing after preprocessing by top-hat transform [2]. ROI is further segmented to avoid the problem of multiple lanes. Segmenting the ROI has the advantage of dividing multiple lanes present in the ROI. This ROI is further divided into left and right sub-regions. Lane marking using HT is carried out in segmented regions of an image. Processing an image without segmentation will detect many Hough lines due to which ambiguity will be created in estimating lane departure. Segmenting ROI will reduce the complexity of the lane detection. Segmentation helps to give lane identification in appropriate manner giving only desired lane lines which are required for estimating lane departure information. This methodology will have the net effect of enhancement in the speed of operation; reduced ambiguity, hence the computational time required for lane departure warning will be reduced. Thus, driver will get lane departure information instantly and will have more warning onset time. It is desirable for LDWS to have more onset time. Onset time is the amount of time the driver gets to bring the car in lane when deviated out of lane. 
The paper is organized as follows. Section 4.1 describes procedure for dynamic threshold value selection. Section 4.2 describes segmentation of ROI. Modified lane departure method is elucidated in section 4.3. Section 5 explains experimental validation. Section 6 concludes the paper.

\section{DYNAMIC THRESHOLd VALUE SELETION}

In this paper, a method based on histogram statistics will be used to determine the fitting threshold value dynamically.

\subsection{Proposed Method}

The procedure is to define a neighborhood and move its center from pixel to pixel. At every location, histogram of the points in the neighborhood is first computed and thereafter the histogram specification transformation function is obtained. This function is then used to map the intensity of the pixel centered in the neighborhood. As shown in Figure 1, the

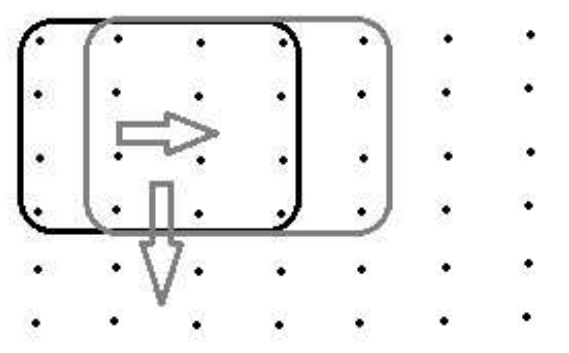

Figure 1. Pixel translation with $4 \times 4$ neighborhood

center of the neighborhood region is then moved to an adjacent pixel location and the procedure is repeated. Because only one row or column of the neighborhood changes during the pixel- to-pixel translation of the neighborhood, updating the histogram obtained in the previous location with the new data introduced at each motion step is possible. Row translation is shown in Figure 1. A $4 \times 4$ neighborhood is taken into consideration. This method has many advantages as can be seen from the Figure. Figure 2 shows the histogram of input and output image. 

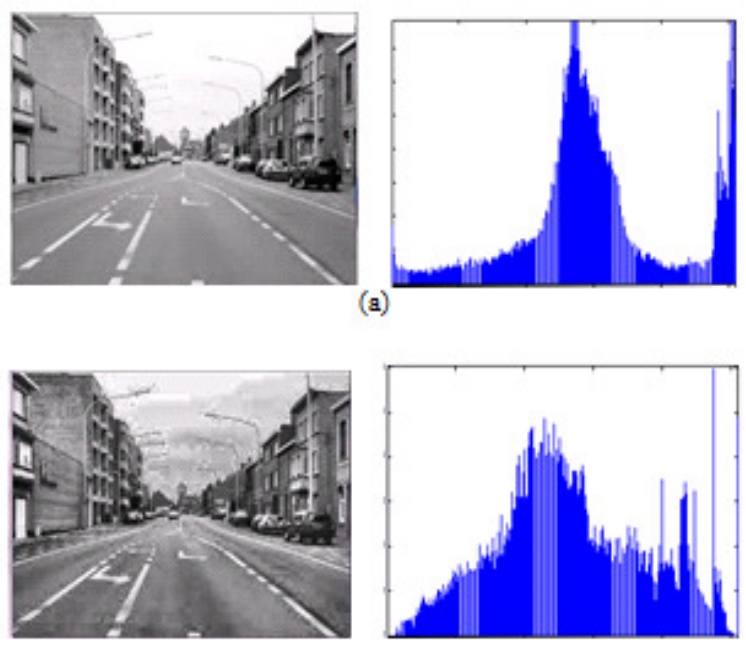

(b)

Figure 2. Histogram Specification Transformation Function. (a) Input Image (b) Output Image

Output image is obtained when the proposed method of dynamic threshold value selection is applied to an input image. Rayleigh distribution is taken into account in histogram specification transformation because it describes the random level brightness and contrast ratio of lane images appropriately. From Figure 2 (b) it is clear that the histogram is equalized and uniformly spaced. This process gives an input image an enhanced contrast level which makes lane detection easier. Figure 3 shows the flow of the algorithm.

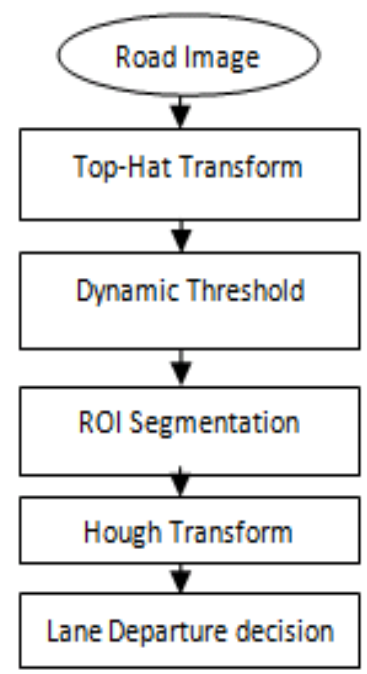

Figure 3. Flow of the algorithm

\subsection{ROI Segmentation}

Lower area of a lane image, shown dotted in Figure 4, is considered as region of interest (ROI). In this part of an image, road lanes are present. This is the lower region of the view seen by a camera which can be situated inside a car near rear view mirror. This ROI is 
further divided into left and right sub- regions. Lane marking using Hough Transform (HT) will be carried out in segmented regions of an image

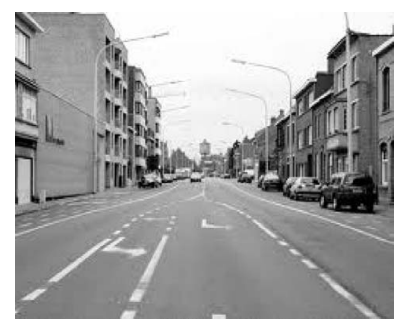

Figure 4. Region of Interest

Segmentation helps to give lane identification in appropriate manner giving only desired lane lines which are required for estimating lane departure information. This methodology will have the net effect of enhancement in the speed of operation. Also, with reduced ambiguity, the computational time required for lane departure warning is reduced. Thus, driver will get lane departure information instantly and will have more warning onset time.

\subsection{Modified Lane Departure Method}

The new proposed methodology for lane departure indication is described in this section. ROI of an image is extracted and represented as $R_{i}$. Edges in an image are detected using Hough transform. Hough origin $\mathrm{H}_{\mathrm{o}}$ is placed at the coordinate $(x / 2,0)$. Edges of lanes are extracted. Left edge mid-point and right edge mid-point viz. $\mathrm{M}_{L}, \mathrm{M}_{R}$ is calculated. A line joining from each mid-point to Hough origin is plotted and its length is measured as $\mathrm{K}_{L}$ , $\mathrm{K}_{R}$. Also, horizontal distance between the mid-points is noted down as length $C$ shown below in Figure 5.

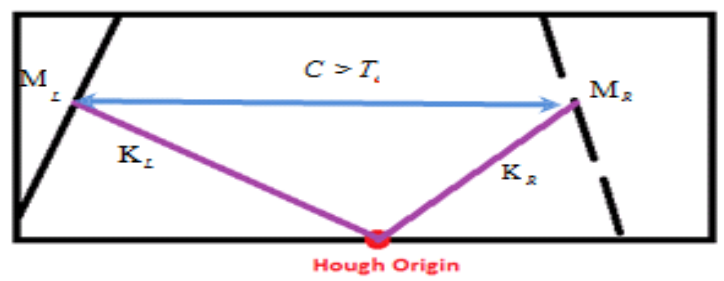

(a)

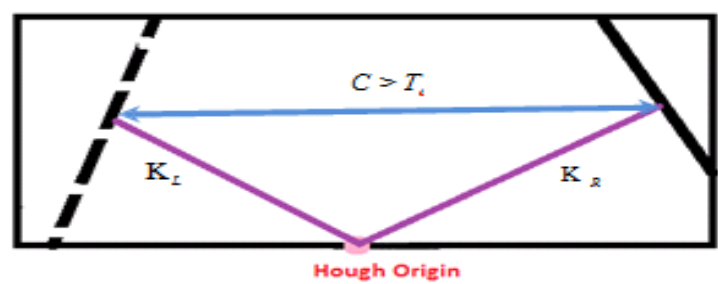

(b)

Figure 5. New Lane Departure Calculation on ROI (a) Left departure, (b) Right departure

If the value of length $C$ is greater than initial threshold value $\mathrm{T}_{i}$ then the position of car will be examined for departure. The terms $\mathrm{K}_{\mathrm{L}}, \mathrm{K}_{\mathrm{R}}$ are used to obtain information in this regard. As shown in above Figure 5 (a), if length $\mathrm{K}_{\mathrm{R}}$ is less than $\mathrm{K}_{\mathrm{L}}$ then car is near right lane otherwise 
if length $\mathrm{K}_{R}$ is greater than $\mathrm{K}_{\mathrm{L}}$ then car is near left lane. The initial thresholds for minimum lengths are set. If either of the length $K_{L}, K_{R}$ reduces below some threshold $T_{L}, T_{R}$ then lane departure on left side or right side occurs and necessary warning will be given to driver. The algorithm for proposed lane departure method is given in following pseudo code

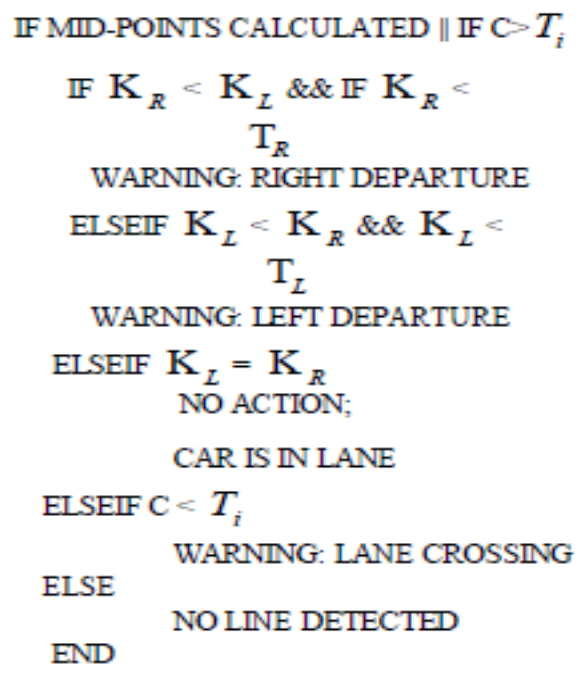

On the contrary, if the value of $C$ is lesser than initial threshold value $\mathrm{T}_{i}$, as shown in Figure 6, car is crossing the lane and is on the central axis of the road

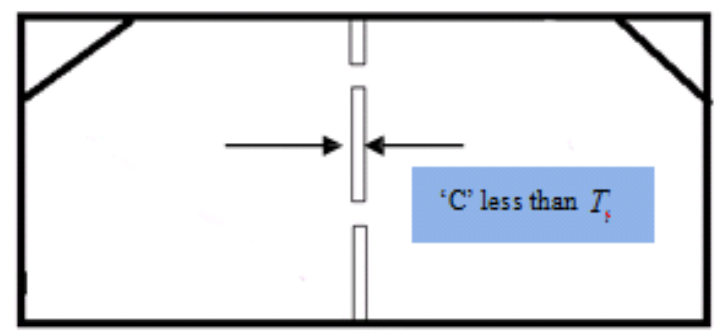

Figure 6. New Lane Departure Calculation on ROI

As shown in this Figure, dotted lane marking is identified. Edges are extracted with outer boundaries. The length $C$ is the distance between the edges shown in Figure 6. $C$ is always less than the initial threshold value in case when car is in left or right lane. Also, during left or right departure, $C$ is always greater than initial threshold. ROI segmentation is taken into account. The uniqueness of the algorithm lies in considering value of $C$ as shown in Figure 5.

Three cases are assumed:

Case I: $C$ is greater than initial threshold value $T_{i}$ when left departure occurs - In this case, the value of $C$ is greater than 50 . The length $\mathrm{K}_{\mathrm{L}}, \mathrm{K}_{\mathrm{R}}$ are calculated. Centroid of $\mathrm{K}_{\mathrm{L}}, \mathrm{K}_{\mathrm{R}}$ is estimated which decides $C$ value. For left departure, $\mathrm{K}_{\mathrm{L}}<\mathrm{K}_{\mathrm{R}}$ is condition is satisfied. 
Case II: $\mathrm{C}$ is greater than initial threshold value $T_{i}$ when right departure occurs - In this case, the value of $C$ is greater than 50 . For left departure, $\mathrm{K}_{\mathrm{R}}<\mathrm{K}_{\mathrm{L}}$ condition is satisfied.

Case III: $C$ is less than initial threshold value $T_{i}$ - In this case $C$ value is less than 50. Car is crossing the lane.

\section{EXPERIMENTAL VALIDATION}

The proposed algorithm of lane departure is simulated in MATLAB. The software runs on i5 processor at $2.53 \mathrm{GHz}$.As shown in Figure 7 (a) original image is shown. The lane detection is performed using Hough transform. The detected lane boundaries are shown in Figure 7 (b) in green color. It seen that HT detects lane boundaries accurately.

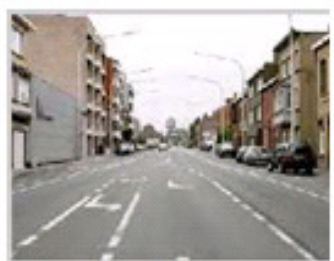

(a)

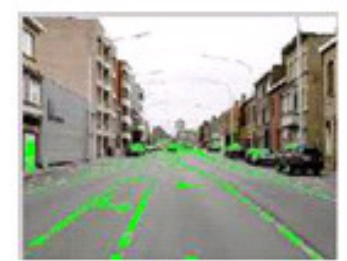

(b)

Figure 7. Lane Detection. (a) Original Image, (b) Lane Detection shown in green color

Modified lane departure method is used to generate warning to the driver. If the car is deviated from the lane, the color of identified lane markings is changed from green to red. A caution or a warning is generated and displayed to the driver. Figure 8 (a) shows that car is departing towards right side. Figure 8 (b) shows that car is crossing the middle boundary and is at the center on a road. Figure 8 (c) shows that car is departing towards left side.



(a)

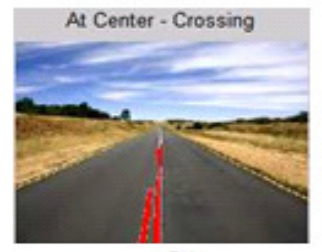

(b)

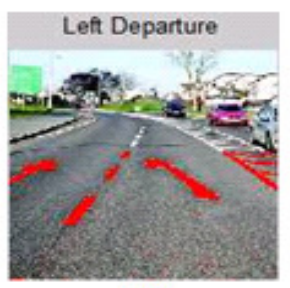

(c)

Figure 8. Lane Departure shown in Red color. (a) Right Side, (b) At Center, (c) Left Side

In case III, $C$ value is 130 . Also, length $\mathrm{K}_{\mathrm{R}}$ is greater than $\mathrm{K}_{\mathrm{L}}$ informing left departure condition has occurred. Thus, accurate predictions are obtained using the proposed algorithm. Table 1 shows that the proposed algorithm gives lane departure information in fraction of second, with average value equal to 0.053622 second. The second last column 
shows time required for each execution of identifying the departure

Table 1: Lane Departure Parameters of Proposed Algorithm

\begin{tabular}{|c|c|c|c|c|c|}
\hline Image & $\mathbf{C}$ & $\mathbf{K}_{\mathbf{L}}$ & $\mathbf{K}_{\mathbf{R}}$ & Time (s) & Departure \\
\hline 1 & 100 & 72 & 58 & 0.051774 & Right \\
\hline 2 & 79 & 95 & 95 & 0.046725 & In Lane \\
\hline 3 & 130 & 68 & 134 & 0.062367 & Left \\
\hline 4 & 120 & 80 & 49 & 0.053745 & Right \\
\hline 5 & 150 & 80 & 80 & 0.053512 & In Lane \\
\hline 6 & 110 & 70 & 138 & 0.054011 & Left \\
\hline 7 & 115 & 69 & 140 & 0.053113 & Left \\
\hline
\end{tabular}

\section{CONCLUSION}

In this paper, an improved method for lane departure warning system is presented. Hough transform is used to detect the lane markings. The lane departure method is improved by ROI segmentation technique. By measuring the distance between the lanes and using it to make out decision for left or right departure, the proposed algorithm accurately detects the lanes in short span of time. It is observed that the proposed algorithm has average execution time of 0.053622 second. It has the benefit of less complexity and fast execution. This algorithm, if optimized, will further enhance the speed of operation. For lane departure warning system it is necessary that the algorithm must be executed in short span of time with better accuracy so that driver will get more onset time to bring the car in lane. Our paper fulfills these conditions by giving less time to generate warning. Thus, proposed algorithm is suitable for real-time application for LDWS.

\section{ACKNOWLEDGEMENTS}

The authors would like to thank everyone, just everyone!

\section{REFERENCES}

[1] National Highway Traffic Safety Administration, http://www.nhtsa.dot.gov/

[2] Long Chen, Qingqyan Li and Qin Zou, "Block-Constraint Line Scanning Method for Lane Detection”, IEEE Intelligent Vehicles Symposium, 2010

[3] Robert M. Haralick and Linda G. Shapiro, "Computer and Robot Vision," Vol.1, Addison Wesley Publishing Company Inc., 1992.

[4] Yue Feng WAN, Francois CABESTAING and Jean-Christophe BURIE, "A new edge detector for Obstacle Detection with a Linear Stereo Vision System”, IEEE Proceedings, 2010, pp. 130 - 135.

[5] Mathias Perrollaz, Anne Spalanzani and Didier Aubert, "Probabilistic representation of the uncertainty of stereo vision and application to obstacle detection", 2010 IEEE Intelligent Vehicles Symposium Univeristy of California, San Diego, Ca, USA, June 21-24 2010, pp.313-318.

[6] C. R. Jung and C. R. Kelber, "A robust linear parabolic model for lane following," Proceedings of XVII Brazilian Symposium on Computer Graphics and Image Processing, Oct. 2004, pp. 7279. 
[7] Joel C. McCall and Mohan M.Trivedi, "Video-based Lane Estimation and Tracking for Driver Assistance: Survey, System, and Evaluation", IEEE Transactions on Intelligent Transportation Systems, vol.7, 2006, pp.20-37, doi: 10.1109/TITS.2006.869595.

[8] Broggi and S. Berte, "Vision-based Road Detection in Automotive Systems: a Real-time Expectation-driven Approach”, Journal of Artificial Intelligence Research, vol.3, 1995, pp. 325-348.

[9] Pei-Yung Hsiao, Chun-Wei Yeh, Shih-Shinh Huang, and Li-Chen Fu, "A Portable Vision-Based Real-Time Lane Departure Warning System: Day and Night", IEEE Transaction on Vehicular Technology, vol. 58, No. 4, May 2009

[10] Bing-Fei Wu, Senior Member, IEEE, Chuan-Tsai Lin, Student Member, IEEE, and Yen-Lin Chen, Member, IEEE, "Dynamic Calibration and Occlusion Handling Algorithms for Lane Tracking", IEEE Transaction on Industrial Electronics, vol. 56, No. 5, May 2009.

[11] Nak Yong Ko, Reid Simmons, Koung Kim, "A Lane based obstacle avoidance Method for Mobile Robot Navigation”, KSME International Journal, Vo. 17, No. 11, pp. 1693-1703, 2010.

\section{Authors:}

Sachin Sharma, Ph.D. pursuing, is Assistant Professor, Electronics and Communication Department, SVBIT, Gandhinagar (Gujarat). He is having more than 5 years of experience in Academics, Research \& Industry. He has published numerous articles related to Image Processing, Digital Signal Processing, and Intelligent Transportation Systems. He is an active member of several professional societies, including ISTE, IEEE and SAE.

Dr. Dharmesh Shah is working as Principal at LCIT, Bhandu (Gujarat). He is

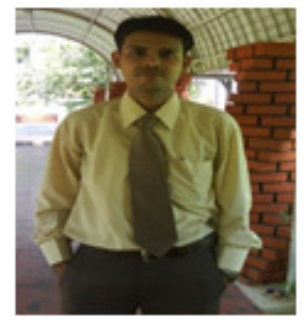
also the Dean - Engineering (Zone II), GTU, Ahmedabad. He is having more than 15 years of experience in Academics, Research \& Industry. He has published numerous articles related to VLSI, Digital Signal Processing, and Image Processing. He is an active member of several professional societies, including IETE, ISTE and IEEE. 